\title{
Une seule ou plusieurs femmes-truies? Une lecture virtualisante de Truismes de Marie Darrieussecq
}

\author{
ALISTAIR ROLLS \& \\ MARIE-LAURE VUAILLE-BARCAN
}

Depuis la parution de Truismes de Marie Darrieussecq en 1996, une approche critique a proliféré selon laquelle l'histoire serait celle d'une métamorphose. La double identité de la protagoniste, la dualité de cette héroïne oscillant entre femme et truie, a été trop séduisante pour que ses lecteurs se demandent si cette pluralité du texte ne serait pas plutôt illusoire. Loin d'une transformation, ce que nous voyons dans ce roman est plutôt la mise en scène d'une activité de mutation sans début ni fin, et où toute la structure textuelle fournie par l'auteure tendrait à capturer sur la page quelque chose de foncièrement a-structuré. Nous nous proposons d'offrir une lecture virtualisante, qui cherche à expliquer l'identité textuelle tout en tenant compte de la nature ouverte, multiple et dynamique du roman. La virtualisation, selon Pierre Lévy, "se présente comme le mouvement même du 'devenir autre' - ou hétérogénèse - de l'humain". ${ }^{1}$ Aussi seront abordées l'identité fluide de la protagoniste $^{2}$ - la façon dont la femme-truie n'est jamais ni femme ni truie, mais mouvement, désir et devenir perpétuels - et l'autoréflexivité du texte qui tend toujours vers l'extérieur ou l'intertexte pour mieux éclairer son contenu. Nous verrons que l'approche du texte littéraire préconisée par Barthes dans "La mort de l'auteur" ou Le Degré zéro de l'écriture, qui nous permettra d'analyser le corps de la protagoniste en tant que désir de lire et d'être lu, c'est-à-dire la force désirante du texte, se révélera proche d'une lecture deleuzienne de la femme-truie comme devenir-animal. Car le devenir de la femme-truie est emblématique selon nous du devenir du texte.

La narratrice prévient ainsi le lecteur dès la première page:

[I]l faut que j'écrive ce livre sans plus tarder, parce que si on me retrouve dans l'état où je suis maintenant, personne ne voudra ni m'écouter ni me croire. Or

\footnotetext{
${ }^{1}$ Pierre Lévy, Qu'est-ce que le virtuel? (Paris: Éditions La Découverte \& Syros, 1998), p. 10.

${ }^{2}$ Par souci de clarté, nous nous permettons d'utiliser le néologisme de la forme féminine.
} 
tenir un stylo me donne de terribles crampes. [...] Je ne vous parle pas de la difficulté pour trouver ce cahier, ni de la boue, qui salit tout, qui dilue l'encre à peine sèche. J'espère que l'éditeur qui aura la patience de déchiffrer cette écriture de cochon voudra bien prendre en considération les efforts terribles que je fais pour écrire le plus lisiblement possible. ${ }^{3}$

Comme dirait Pierre Lévy, c'est comme si elle inventait l'écriture: "Loin d'anéantir le texte, la virtualisation semble le faire coïncider à son essence soudain dévoilée. Comme si la virtualisation contemporaine accomplissait le devenir du texte. Comme si nous sortions d'une certaine préhistoire et que l'aventure du texte commençait vraiment. Comme si nous venions, enfin, d'inventer l'écriture."4

Comprendre, dans ce contexte de virtualisation, signifie ouvrir le texte, le rendre fluide; son devenir nécessite le rôle du lecteur, car l'essence du texte ne doit pas se confondre avec le produit qu'est l'œuvre. Nous dirons de la femmetruie que - en tant que force désirante - elle manifeste deux désirs qui à première vue semblent être en relation binaire, c'est-dire opposée: d'une part elle cherche à actualiser le texte, c'est-à-dire à en faire une œuvre lisible; et d'autre part elle virtualise son identité textuelle, dissolvant le texte dans l'intertexte et la diversité créative du scriptible. Pourtant, il ne faut pas voir dans ces désirs, qui sont dans Truismes parallèles et simultanés, une contradiction: cette différence produit le livre-rhizome. Truismes est à la fois œuvre et texte; il est déjà produit et toujours en voie de production. C'est dans ce sens que nous comprendrons le devenir de la femme-truie: désir multiple et non pas binaire.

Tous les problèmes de la protagoniste s'expliquent par et dans la virtualisation. En s'offrant un nouveau corps, la femme-truie cherche à alléger les "crampes" du devenir. Comme l'explique Lévy, “en se virtualisant le corps se multiplie. Nous nous créons des organismes virtuels qui enrichissent notre univers sensible sans nous imposer la douleur". ${ }^{5}$ Elle est donc virtualisation par son identité fluide qui, chaque fois qu'elle est près de se concrétiser, s'éloigne ou se perd dans divers espaces liminaires du texte; et elle est actualisation, car elle manifeste toujours désir de fixité et recherche de forme, qu'elle soit humaine ou porcine: "La virtualisation du corps n'est $[\ldots]$ pas une désincarnation mais une réinvention, une réincarnation, une multiplication, une vectorisation, une hétérogenèse de l'humain. Cependant, la limite n'est jamais définitivement tracée entre l'hétérogenèse et l'aliénation, l'actualisation et la réification marchande, la virtualisation et l'amputation." ${ }^{6}$ Aliénation, réification et amputation, exploitation, censure, mise à mort, de l'animal comme de l'humain, sont des thèmes récurrents de Truismes. Marie Darrieussecq place judicieusement en exergue une citation du Norvégien Knut Hamsun sur l'abattage d'un verrat:

\footnotetext{
${ }^{3}$ Marie Darrieussecq, Truismes (Paris: P.O.L., 1996), p. 11. (Édition citée: Folio, 1999.)

${ }^{4}$ Lévy, p. $48 . \quad \quad \quad{ }^{5}$ Lévy, p. $31 . \quad{ }^{6}$ Lévy, p. 31.
} 
Puis le couteau s'enfonce. Le valet lui donne deux petites poussées pour lui faire traverser la couenne, après quoi, c'est comme si la longue lame fondait en s'enfonçant jusqu'au manche à travers la graisse du cou. D'abord le verrat ne se rend compte de rien, il reste allongé quelques secondes à réfléchir un peu. Si! Il comprend alors qu'on le tue et hurle en cris étouffés jusqu'à ce qu'il n'en puisse plus. ${ }^{7}$

Notre analyse étant avant tout d'ordre textuel, nous nous ne arrêterons pas ici sur le passé nazi de cet auteur, que Marie Darrieussecq bien évidemment connaissait, laquelle se livre aussi dans le roman à la dénonciation par la caricature d'un état fasciste. Cette citation est surtout, comme elle l'indique elle-même, "une phrase [...] qui disait bien cet éclair de conscience dans l'esprit du cochon qui va mourir [...] C'est sur l'animal pensant, un paradoxe."' Dès l'exergue donc, par cette référence à l'animal pensant, animalité et nature humaine sont inextricablement mêlées, comme elles le sont dans la perspective deleuzienne, résumée ainsi par la philosophe Éliane Martin-Haag:

Croire en une nature humaine, que la tradition philosophique s'attache à distinguer de l'animalité par la raison, le langage, la perfectibilité, l'histoire, l'espèce ou l'organisme, c'est toujours enfermer l'homme dans une forme ou une logique identitaires, dans un "pli" infernal que l'on ne peut plus défaire. [...] C'est donc dans la création artistique que Deleuze recherche les lignes de fuite et les pointes de déterritorialisation qui passent par un deveniranimal, pour défaire l'organisme humain. ${ }^{9}$

De la même façon que la peinture de Bacon, à laquelle Deleuze a consacré une analyse, remet en cause la perception du corps en présentant l'humain dans les postures les plus animales, la narratrice de Truismes s'affranchit du poids du champ social et familial et de ses truismes (au sens des évidences ou des discours établis) par une série de "dé-formations" corporelles multiples. La jeune prostituée qui s'ignore se libère par le devenir-animal, "la femme étant", comme le dit Éliane Martin-Haag, "toujours réduite à un objet ou à une nature proche de l'animal dans le contrat des société patriarcales". ${ }^{10}$ Et c'est pourquoi, pendant tout le livre, "les dispositifs de pouvoirs tentent de capturer ou de ligaturer"11 ces dispositifs auxquels se joint la mère, tentent de récupérer ou d'abattre celle qui cherche à s'affranchir par le devenir-animal, en traçant maladroitement sur son cahier des lignes de fuite ou de devenir.

\footnotetext{
${ }^{7}$ Darrieussecq, p. 7.

8 Jeannette Gaudet, “'Des livres sur la liberté': Conversation avec Marie Darrieussecq”, Dalhousie French Studies, 59 (2002), 108-118 (p. 113).

${ }^{9}$ Éliane Martin-Haag, "Le devenir-animal et la question du politique chez Gilles Deleuze", p. 1. http://w3.univ-tlse2.fr/philo/IMG/pdf/_MARTIN-HAAG_Le_devenir-animal_et_la_ question_du_politique_chez_Gilles_Deleuze.pdf (informations téléchargées le 4 juillet 2007).

${ }^{10}$ Martin-Haag, p. 8.
} 


\section{Alistair Rolls \& Marie-Laure Vuaille-Barcan}

Ce qui fait du personnage comme du texte une entité infiniment riche en contradictions. Écrivant / lisant, la femme-truie s'actualise à partir de données intertextuelles qui lui préexistent et se dote d'un corps qui se virtualise constamment; écrite / lue, elle est réifiée et, comme le verrat dans la citation en exergue de l'épigraphe, menacée de finir découpée. Elle est donc à la fois mise à mort et (re)naissance.

Il ne s'agit donc pas d'un roman de transformation. Si nous suivons les modèles de la duplication et de l'effacement du sujet offerts par Mireille Buydens dans son essai sur l'approche deleuzienne d'Internet et que nous les appliquions à notre femme-truie virtuelle, nous constatons que le désir d'un "moi" concret mènerait à une métamorphose, en truie et en femme, alors que la dissolution de cette identité qu'on trouve dans le sujet postmoderne ou dans la jouissance du texte scriptible constituerait plutôt une anamorphose, c'est-à-dire le "dépassement de la forme vers l'intensité aformelle". ${ }^{12}$ Comme l'explique Buydens:

Ce qui est assuré en dernière instance par cette indexation négative du concept de forme n'est autre que la plus grande fluidité, c'est-à-dire la plus grande liberté, de la création conceptuelle : si aucune forme n'est donnée a priori [...] il s'ensuit que l'agencement provisoire de singularités mouvantes et libres et que l'homme ou le hasard sont libres de les réassembler autrement. ${ }^{13}$

Si Truismes est l'histoire d'une identité anamorphique et multiple, c'est que le texte se dissout, volontairement, dans l'extériorité de l'intertexte, avant même notre première rencontre avec la femme-truie. Nous savons que, depuis la mort de l'auteur, la forme du texte est créée et dépassée chaque fois qu'est effectuée une lecture. Dans le même temps qu'il est actualisé, rendu œuvre à partir d'un concept ou une idée d'auteur, le texte est aussi virtualisé: ce qui existe sous forme de mots est lu et doté, en raison de cette lecture, par un nombre quasi infini de significations.

En se référant à Jacques Derrida, Gary Gutting explique qu'“écrire révèle les particularités et limitations essentielles de la pensée humaine." ${ }^{14}$ La trace que laisse l'auteur sur la page n'est jamais qu'un morceau de chair - idée devenue texte - qui se laissera à son tour dépecer, manger, apprécier, chanter et imaginer par le lecteur. Nous suggérons que devenir truie est devenir texte dans un espace particulier où "se délimiter" signifierait passer toujours au-delà de ses limites. Ce

\footnotetext{
${ }^{11}$ Martin-Haag, p. 3.

${ }^{12}$ Mireille Buydens, "La Forme Dévorée: Pour une approche deleuzienne d'Internet" in Thierry Lenain (ed.) L'Image: Deleuze, Foucault, Lyotard (Paris: Librairie Philosophique J. Vrin, 1998), pp. 41-63 (p. 53).

${ }^{13}$ Buydens, p. 53.

${ }^{14}$ C'est nous qui traduisons: "[W]riting reveals the essential peculiarities and limitations of human thought." Gary Gutting, French Philosophy in the Twentieth Century (Cambridge: Cambridge University Press, 2001), p. 291.
} 
sont donc les limites de l'espace textuel, la structure a-structurée de la multiplicité, qui est repensée dans le devenir de la femme-truie.

Par conséquent, nous remettons en cause la principale opposition sur laquelle Truismes est fondé: l'homme n'est ni le contraire ni le revers du cochon. Si le terme "cochon" s'applique si souvent à des comportements humains, c'est qu'il y a autant de similarités que de différences entre ces deux espèces. L'impossibilité de concevoir une opposition femme-truie est soulignée par d'autres cas de "transformation": l'amant Yvan qui devient loup, l'homme politique Edgar qui devient cheval, etc. D'ailleurs, quand un verrat ou un loup atteint chez Darrieussecq l'apogée de son être animal, c'est pour devenir séduisant dans sa "virilité": "Yvan était gris argenté, avec un long museau à la fois solide et très fin, une gueule virile, forte, élégante, de longues pattes bien recouvertes et une poitrine très large, velue et douce. Yvan, c'était l'incarnation de la beauté."15 Le lecteur se trouve face à une confusion de catégories sans qu'il y ait véritablement de zone grise: la femme-truie n'est pas moitié femme, moitié truie. Femme et truie feront ainsi rhizome précisément dans la mesure où elles ne sont pas la même. Cette idée deleuzienne de l'être, où répétition est démonstration de différence, peut nous rappeler la différence du texte dans les analyses de Roland Barthes, que Barbara Johnson décrit de la manière suivante: "[1]a différence d'un texte ne s'explique pas par son identité unique ou spéciale; c'est sa façon d'être différent de lui-même". ${ }^{16}$ Femme et truie sont la répétition d'une même identité; ni unité, ni pluralité, la femme-truie est un système complexe de connexions faites entre des entités différentes.

Par des lignes de fuites le lecteur saute à l'intérieur d'un même texte dans d'autres textes. Cette intertextualité constitue le devenir-loup du texte: "Malheureusement le Pont n'a jamais été terminé, il n'y avait qu'Yvan les soirs de pleine Lune qui pouvait d'un bond assez puissant rejoindre la rive, qu'est-ce qu'il était fort Yvan."17

Dans l'architecture moderne, qui cherche à maximiser l'intégration de la demeure de l'homme dans son environnement, un saut de loup est un dispositif permettant d'introduire du jour dans des pièces sans fenêtre; le saut-de-loup Heliobus, par exemple, "[transforme] de sombres recoins, débarras et dépôts en des espaces baignés de lumière naturelle qui peuvent servir à de multiples usages". ${ }^{18}$ Autrement dit, un saut de loup permet à l'extérieur de se confondre avec l'intérieur

\footnotetext{
${ }^{15}$ Darrieussecq, p. 120.

16 "[A] text's difference is not its uniqueness, its special identity. It is the text's way of differing from itself." Barbara Johnson, "The Critical Difference" in Diana Knight (ed.) Critical Essays on Roland Barthes (New York: G.K. Hall \& Co., 2000), pp. 174-182 (p. 175). Cf. Gary Gutting sur la différence chez Deleuze : "[But] if to be is to be different, what could repetition be other than an expression of a being's difference with itself?" (p. 336).

${ }^{17}$ Darrieussecq, p. 130.

${ }^{18} \mathrm{http}: / /$ www.heliobus.com/pages_f/spiegelschacht.html (informations téléchargées le 27 octobre 2006).
} 


\section{Alistair Rolls \& Marie-Laure Vuaille-Barcan}

d'une structure, ce à quoi il servait autrefois dans le cadre de l'architecture des grands jardins français où il "[permettait] de conserver une continuité visuelle entre le jardin et l'horizon extérieur". ${ }^{19}$ Ainsi, lorsque Yvan le loup-garou de Truismes de Marie Darrieussecq se révèle capable de franchir d'un bond la Seine à l'endroit où le Pont-Neuf tombe en ruines, il apporte au texte les qualités du saut de loup: il fait se confondre les espaces intérieurs et extérieurs jusqu'à ce qu'il ne puisse plus s'agir de deux espaces séparés, ni même d'un espace réuni. Aussi vaut-il mieux que l'on conçoive ici l'espace architectural et textuel comme de l'espace. La figure du saut de loup nous rappelle que le loup lui-même, encore qu'il puisse exister seul, puise son identité dans une multiplicité dont il fait partie mais qui demeure en dehors de son corps proprement dit. Selon Gilles Deleuze et Félix Guattari, il s'agit là de son devenir-animal, devenir qui veut qu'on ait 'toujours affaire à une meute, à une bande, à une population, bref à une multiplicité". ${ }^{20} \mathrm{Il}$ en va de même pour le texte: quoiqu'on puisse en toute logique parler d'un texte littéraire, en tant qu'assemblage de mots, lignes et paragraphes, le tout délimité par un début et une fin, ce texte ne peut exister autrement qu'en relation avec d'autres textes qui, tout en existant en dehors de notre texte littéraire, l'entourent, le traversent et entrent en relation avec lui, si bien que l'on finit par perdre de vue ces mêmes barrières structurales qui nous permettaient d'appréhender notre texte au singulier. Ce qui nous reste est $d u$ texte, ou ce que Julia Kristeva dénomme "l'intertexte", terme singulier qui incorpore une entité ni singulière, ni plurielle, mais multiple. ${ }^{21}$ On pourrait se demander ce que serait un texte tout seul; l'intertexte, en revanche, ressemble au lupulement: "Qu'est-ce qu'un loup tout seul? et une baleine, un pou, un rat, une mouche? [...] Le loup n'est pas d'abord un caractère ou un certain nombre de caractères, c'est un lupulement. Le pou est poululement". ${ }^{22}$

Quand on aborde le texte scriptible - c'est-à-dire que l'on poursuit une lecture active du texte - on fait pour ainsi dire un saut de loup; on actionne les liens existant entre le texte qu'on lit et tous ces textes qui font rhizome avec lui. Le devenir-animal d'un texte n'est autre que son intertextualité, cette capacité à exister à la fois dans ces propres pages et dans les pages d'autres textes: "Nous ne devenons pas animal sans une fascination pour la meute, pour la multiplicité. Fascination du dehors? Ou bien la multiplicité qui nous fascine est-elle déjà en rapport avec une multiplicité qui nous habite au-dedans?" ${ }^{23}$ Comme nous le rappelle Pierre Lévy, exister signifie, selon l'étymologie, à la fois être placé et hors de, comme si pour exister il fallait être là et dans le même temps sortir de là. ${ }^{24}$ L'acte d'écrire un texte

\footnotetext{
${ }^{19} \mathrm{http} / / / \mathrm{www}$. coutaubegarie.com/php/chateaux_photo.php (informations téléchargées le 27 octobre 2006).

${ }^{20}$ Gilles Deleuze et Frédéric Guattari, Mille Plateaux: Capitalisme et schizophrénie 2 (Paris: Les Éditions de Minuit, 1980), p. 292.

${ }^{21}$ Cf. Julia Kristeva, Semeiotikè: recherches pour une sémanalyse (Paris: Points, 1969).

${ }^{22}$ Deleuze et Guattari, p. $293 . \quad{ }^{23}$ Deleuze et Guattari, p. 293.

${ }^{24}$ Lévy, p. 18.
} 
consisterait alors à le faire exister concrètement et le faire sortir d'ailleurs, c'est-àdire à le déterritorialiser. A en croire Deleuze et Guattari, cet acte de production du texte comprend un devenir qui va plus loin que la simple écriture: "Si l'écrivain est un sorcier, c'est parce qu'écrire est un devenir, écrire est traversé d'étranges devenirs qui ne sont pas des devenirs-écrivain, mais des devenirs-rat, des devenirs-insecte, des devenirs-loup, etc." ${ }^{25}$ C'est pourquoi il serait illogique de se demander si la protagoniste de Truismes devient truie parce qu'elle est victime des cochonneries des hommes ou si la jeune fille devient cochonne parce que déjà truie. Le devenirtruie de la protagoniste fait partie intégrante de la production du texte. Ce roman dit de transformation, puisque dans sa traduction anglaise il s'agit de Pig Tales: A Novel of Lust and Transformation, ${ }^{26}$ est pour nous bien davantage un roman du devenir du texte, la mise en scène du devenir-animal du texte en création.

Le texte de la femme-truie exprime son trouble dès les premières lignes, mais le désir d'écrire se montre plus fort que tout autre raisonnement:

Je sais à quel point cette histoire pourra semer de trouble et d'angoisse, à quel point elle perturbera de gens. [...] Mais il faut que j'écrive ce livre sans plus tarder, parce que si on me retrouve dans l'état où je suis maintenant, personne ne voudra ni m'écouter ni me croire. ${ }^{27}$

Quel serait donc cet état dans lequel notre écrivaine se trouve? On ne peut savoir s'il s'agit de femme ou de cochon; la seule chose dont on puisse être sûr, c'est que le texte s'écrie (s'écrit). Et là encore, on pourrait suggérer que le texte préexiste à ces aveux. En effet, l'acte d'écriture annoncé ici est le même que celui qu'effectue un lecteur comme Roland Barthes face au texte scriptible. C'est-à-dire que la femmetruie, en tant que devenir-écrivain, est d'abord lectrice. Et ce qu'elle lit est le texte qui préexiste au sien, quel qu'il soit; en l'occurrence, l'épigraphe tirée de Benoni de Knut Hamsun.

Comme nous l'avons déjà constaté, il ne s'agit pas dans Truismes d'une transformation, pour la simple raison qu'il n'y a pas de progression linéaire depuis un point fixe que l'on peut nommer 'début', jusqu'à un point ultime où la transformation serait menée à son terme. Il en va de même pour l'extrait de Benoni où est décrit un acte qui est déjà en cours. Cet acte - l'abattage d'un verrat - n'a ni début, ni à vrai dire de fin car n'est décrit dans l'épigraphe que l'épuisement des cris de la bête.

\footnotetext{
${ }^{25}$ Deleuze et Guattari, pp. 293-294.

${ }^{26}$ Le titre de la version anglaise de Truismes, traduite par Linda Coverdale, contient un soustitre selon lequel il s'agirait dans le texte de désir et de transformation: Pig Tales: A Novel of Lust and Transformation. Alors que nous sommes d'accord pour y trouver du désir, sexuel et textuel, nous préférons parler d'anamorphose que de métamorphose, de devenir plutôt que de transformation.

${ }^{27}$ Darrieussecq, p. 9.
} 
L'impact de l'épigraphe sur le lecteur de Truismes est de faire comprendre que l'histoire se déroule déjà. L'abattage du verrat résume aussi l'acte de lire le texte scriptible ou de faire du scriptible à partir du lisible: tout lecteur anime et donne vie au texte dans le même temps qu'il l'épuise et l'achève. Si le devenir d'un texte est d'être lu, il s'ensuit que ce dernier est également destiné à naître et mourir encore et toujours. Et comme nous l'indique la femme-truie, lire est un acte qui "[sème] trouble et angoisse". ${ }^{28}$ L'épigraphe offre à Truismes une enveloppe protectrice (une couenne) dure, que la protagoniste commente, et que le lecteur a du mal à traverser; après la perforation initiale, cependant, la lame du lecteur s'enfonce aisément et on lit les horreurs de l'histoire plus facilement, et c'est à ce moment que le cri du texte (re)commence à vivre son épuisement.

L'épigraphe met donc en scène la lecture du texte scriptible, à savoir l'écriture de la femme-truie, qui n'est rendue possible que par le devenir-animal de la protagoniste dont l'histoire est celle des mises en relation de l'intertexte. Pour lire Hamsun, la femme-truie actualise et déterritorialise la difficulté de passer par la couenne, ce qu'elle présente sous forme d'écriture. Avec son passage dans la chair tendre, commence l'histoire proprement dite; et ici la mise en scène montre la perversité de la lecture:

Le directeur de la chaîne m'avait prise sur ses genoux et me tripotait le sein droit, et le trouvait visiblement d'une élasticité merveilleuse. [...] Le directeur de la chaîne tenait mon sein droit dans une main, le contrat dans l'autre main. [...] Ses doigts étaient descendus un peu plus bas et déboutonnaient ce qu'il y avait à déboutonner, et pour cela le directeur de chaîne avait été bien obligé de poser le contrat sur le bureau. Je lisais et relisais le contrat par-dessus son épaule $[\ldots]$ Le directeur de la parfumerie m'avait fait mettre à genoux devant lui et pendant que je m'acquittais de ma besogne je songeais à [ces] produits de beauté... ${ }^{29}$

Dans cette scène, la première pour le lecteur de Truismes où la femme-truie est présentée sous la forme d'une femme, on trouve une structure perverse qui semblerait à l'origine des cochonneries du texte: le corps de la femme-truie est ici espace de jouissance ; en incarnant la fellation, elle est à la fois active (donne du plaisir), passive (se laisse devenir site de plaisir) et neutre (alors qu'elle ne paraît pas y prendre plaisir, elle n'en est pas non plus gênée). Mais la jouissance que nous lisons ici est autoréflexive dans la mesure où elle est celle de la lecture elle-même, de la production du texte scriptible. Bien qu'elle soit chez le directeur soi-disant pour signer un document, pour fixer son moi sous forme de contrat écrit, elle préfère lire et se laisser lire: en lisant le contrat elle abandonne son moi: elle abandonne sa conscience dans la rêverie et laisse toute possibilité de formation de beauté et

\footnotetext{
${ }^{28}$ Darrieussecq, p. 9.

${ }^{29}$ Darrieussecq, pp. 11-12.
} 
d'identité réelles se faire remplacer par une idée de Beauté abstraite. Ce qui reste entre les mains du directeur est un corps à l'état pur. ${ }^{30}$ Ainsi devient-elle texte à lire. Cependant, on ne peut nier qu'elle est elle-même en train de lire. La jouissance est double, allant dans les deux sens: en se prononçant texte, elle se fait un moi de jouissance et d'abandon ; en lisant, elle nous rappelle le désir du texte, qui est non seulement celui que la lecture offre au lecteur mais aussi celui qui est exercé sur le lecteur. Comme la jouissance est "créatrice, dynamique, fluide et transgressive", ${ }^{31}$ la femme-truie ne se montre ni femme, ni truie; elle est texte et désir.

Selon le schéma de Barthes, une dialectique se forme selon laquelle, au moment où il crée le texte, le lecteur se fait créer par lui. ${ }^{32}$ Pour que Truismes puisse fonctionner intertextuellement, il n'est pas suffisant que la femme-truie lise. Il faut aussi qu'elle soit lue. Ici le directeur est à la fois l'auteur, qui se fait obéir par sa protagoniste, et le lecteur, qui la désire et fait en sorte que ses gestes soient interprétés. Autrement dit, il n'y a plus d'auteur ni de lecteur mais directeur anonyme ou Autre hétérogène, incarnation du désir de la femme-truie de s'exprimer. On peut aller jusqu'à dire qu'il ne s'agit même pas ici de recherche de forme ni de dissolution de forme; c'est un mouvement et une connexion qui sont présentés.

On n'a plus une tripartition entre un champ de réalité, le monde, un champ de représentation, le livre, et un champ de subjectivité, l'auteur. Mais un agencement met en connexion certaines multiplicités prises dans chacun de ces ordres, si bien qu'un livre n'a pas sa suite dans le livre suivant, ni son objet dans le monde, ni son sujet dans un ou plusieurs auteurs. Bref, il nous semble que l'écriture ne se fera jamais assez au nom d'un dehors. Le livre, agencement avec le dehors, contre le livre-image du monde. Un livrerhizome, et non plus dichotome, pivotant ou fasciculé. ${ }^{33}$

Tandis que nous lecteurs cherchons à y voir la tripartition dont Deleuze et Guattrari parlent dans Mille Plateaux, nous nous rendons compte que ce désir de forme est le même geste vain qui pousse la femme-truie à tracer son "écriture de cochon" dans la boue ${ }^{34}$ et que l'important dans la lecture-fellation est qu'il y ait agencement. Au lieu de représenter le monde, Truismes, en tant que livre-rhizome, offre des possibilités de s'y connecter. En fin de compte, celui qui y voit le monde ne voit traduire que son désir de forme.

Mais comment dire que le monde n'est pas présent dans Truismes? Quand la protagoniste s'offre "un après-midi à l'Aqualand", on ne peut pas ne pas penser

${ }^{30}$ De la même manière, dans la scène célèbre de mauvaise foi de L'Être et le néant, une femme se laisse séduire par son amant en lui abandonnant sa main, ce qui lui permet de nier toute responsabilité dans le jeu de séduction.

${ }^{31}$ Cette description que nous traduisons ici est offerte par Patrick Fuery dans son livre Theories of Desire (Melbourne: Melbourne University Press, 1995), p. 70.

${ }^{32}$ Cf. Fuery, p. $71 . \quad{ }^{33}$ Deleuze et Guattari, p. 34.

${ }^{34}$ Darrieussecq, p. 10. 
à l'Aquaboulevard, espace commercial et centre de loisirs situés sur le boulevard périphérique à la jonction du $\mathrm{XV}^{\mathrm{e}}$ arrondissement et Issy-les-Moulineaux. ${ }^{35}$ Même dans la réalité cet endroit fonctionne comme agencement, car c'est là que s'accomplit la transition entre Paris et la proche banlieue. Dans le roman, il s'agit d'un endroit quasi onirique, où luxe et vice se côtoient:

Il pleuvait dehors mais à l'Aqualand il fait toujours beau et chaud. Aller à l'Aqualand représentait presque un dixième de ma pension d'insertion mensuelle et ma mère n'a pas du tout été d'accord. Elle a même refusé de me donner un ticket de métro et j'ai été obligée, pour franchir la barrière, de me coller contre un monsieur. Il y en a toujours qui attendent les jeunes filles aux barrières du métro. J'ai bien senti que je faisais de l'effet au monsieur; pour tout dire, beaucoup plus d'effet que je n'en faisais d'habitude. Il a fallu, dans les salons de déshabillage de l'Aqualand, que je lave discrètement ma jupe. $^{36}$

On ne saurait dire de cet extrait qu'elle met en scène le vrai Aquaboulevard, ni qu'il s'agit ici d'une femme qui se transforme en truie. Le devenir-truie de la protagoniste se lit déjà dans ses cuisses "roses et fermes" ainsi que dans l'écriture de cochon dont cette description fait partie. Comme la guêpe et l'orchidée font rhizome chez Deleuze et Guattari, la protagoniste se met en mouvement par l'intermédiaire d'un être hétérogène, à savoir "un monsieur". Les deux personnages se réunissent, font rhizom; elle manifeste son devenir-monsieur, et lui son devenir-jeune fille, sans pour autant qu'il y ait transformation: il ne devient pas jeune fille et elle ne devient pas monsieur. Mais dans cette connexion, tous deux expriment un devenir-cochon, dont l'effet jouissif représente le moi multiple d'une femme-truie pour laquelle le fait de "franchir la barrière" ne représente pas son passage à une nouvelle identité mais la fluidité d'une identité rhizomique et périphérique déjà, toujours et omniprésente.

Cathy Cole, dans son analyse du roman policier, signale comment les écrivaines décrivent les villes de manière à ce que la forme qu'elles composent coïncide avec leur vie, si bien que c'est leur identité même qu'elles forgeraient dans les rues du texte. En citant Drusilla Modjeska, elle relate comment "[1]es femmes savent vivre à la périphérie [...] et que nos souvenirs et nos histoires se forment dans le mouvement - entre intérieur et extérieur, passé et avenir, entre l'environnement physique et la société humaine". ${ }^{37}$ Cet espace entre zones de différence, entre êtres hétérogènes, entre auteur(e) et lecteur, est celui de la femme-truie. Elle crée un moi liminaire qu'elle pose comme identité lue et lisante. Aussi le directeur et le monsieur sont-ils des masques derrière lesquels le lecteur

\footnotetext{
${ }^{35}$ Darrieussecq, p. 12. En effet, on trouve à l'Aquaboulevard la même baleine dont parle la femme-truie, à l'exception près qu'elle est en plastique et non pas empaillée.

${ }^{36}$ Darrieussecq, pp. $12-13$.

${ }^{37}$ Cathy Cole, Private Dicks and Feisty Chicks: An Interrogation of Crime Fiction (Fremantle: Curtin University Books, 2004), p. 190, notre traduction.
} 
de Truismes désire la mutation ainsi offerte; en lisant la séduction de la femmetruie il veut lui-même voir et participer, se créer un moi qui lit pour le perdre dans l'abandon du moi qui est lu. Mais à la différence du flâneur, dont on dira qu'il est à la fois voyeur et acteur dans les rues de Paris, oscillant entre ces deux pôles, la femme-truie n'est qu'oscillation, n'occupant aucune des formes esquissées dans le texte. ${ }^{38}$ N'était-ce le désir de fixité qui est sous-jacent à toutes ces lignes de fuite, la femme-truie aurait la liberté de "la création conceptuelle" que Mireille Buydens décrit de la manière suivante: "[S]i aucune forme n'est donnée a priori (puisque le transcendantal est préindividuel, c'est-à-dire $a$-formel), il s'ensuit que toute forme n'est que l'agencement provisoire de singularités mouvantes et libres et que l'homme ou le hasard sont libres de les réassembler autrement." ${ }^{39}$

Pourtant, le roman n'est pas sans forme. En effet, toute l'histoire est aussi celle de la mort de la créativité. ${ }^{40}$ Le verrat, sur la mort duquel le roman est fondé, laisse entendre sa voix chaque fois que la femme-truie est liée aux histoires du royaume des animaux, à l'infini de la tradition orale. Bien qu'elle se dissolve dans ces histoires externes, prenant le saut de loup dans l'intertexte, il n'empêche qu'elle fixe la forme de Truismes. Acte pervers, elle cherche à former l'a-formel. Là où la créativité littéraire (ou texte scriptible) est un devenir continu, l'œuvre (ou le lisible), laquelle a une forme précise, fait ligne morte. L'histoire de notre femme-truie est celle d'un désir créateur rendu œuvre, tracé dans la boue du produit littéraire et non pas de la production:

Car la ligne est "antérieure" aux dessins qu'elle noue: elle ne s'y investit qu'à moitié, ne les inscrit que pour les fuir aussitôt. Capturer la ligne dans le contour qu'elle trace serait la cristalliser en son contraire: la forme, une ligne morte. Aussi la ligne suppose-t-elle, comme ce qui constitue sa vie même, d'être rompue, brisée, connectée sans répit ni préméditation. La figure linéaire (dont le pli ou la multiplicité constitue le concept théorique) se doit d'être asignifiante, car si la figure tracée se voit dotée d'une signification, elle est de ce fait cristallisée, arrimée dans le message qu'elle est censée avoir exprimé. La signifiance, comme la forme substantielle, est une façon d'éterniser, de transformer le précaire en signe conservable. ${ }^{41}$

A différents moments, elle trouvera Benoni, le lira, ne le nommera pas, l'abandonnera à l'oubli et l'abattra, laissant le corps mutilé de ce livre s'attacher à jamais au

\footnotetext{
${ }^{38}$ Non seulement Truismes déforme la figure de la flânerie, mais les plus célèbres des auteurs de Paris sont aussi transformés. C'est le cas d'Honoré de Balzac dont La Comédie humaine se voit décentralisée, mutée dans la banlieue, et tournée en dérision: Honoré, copain de la femmetruie, joue une comédie porcine et vulgaire.

${ }^{39}$ Buydens, p. 53.

${ }^{40}$ Pour nous, le symbole clé de la mort de la créativité est ce moment où l'écrivain achève son œuvre, lui donnant ainsi sa forme finale, celle que liront ses lecteurs.

${ }^{41}$ Buydens, p. 54-55.
} 
sien dans l'espace (inter)textuel de Truismes. Malgré ces tentatives de s'emparer des œuvres d'autrui, elle n'est pas Benoni, et la femme-truie ne sera que pli dans l'intertexte.

Avant qu'il ne soit abattu, comme le verrat de Hamsun et l'auteur de Barthes, Yvan le loup-garou montre à la femme-truie comment "adapter [son] rythme aux fluctuations de la Lune". ${ }^{42}$ Cette maîtrise lui permettrait de rester l'auteure de sa propre transformation. Toutefois, il réussit mieux qu'elle ce mouvement entre les pôles de l'identité plurielle dans la mesure où son rôle dans le roman est d'incarner la force actualisante de l'auteur. Au demeurant, cette différence de niveaux de contrôle importe peu à la femme-truie, qui profite de leur nid au sein de l'“autarcie communautaire" pour subvenir à leurs besoins par la voie d'Internet..$^{43}$ Le paradoxe d'une existence supposée autonome mais qui dépend d'un réseau virtuel où tous seraient inéluctablement interconnectés fait pendant à certains plis dans l'intertexte qui laissent émerger d'autres textes clés de la littérature française. En voyage, par exemple, leur intimité rappelle au lecteur un autre cas d'intériorité percé par le monde de dehors: "[Mais] c'était très excitant de rester ainsi enfermés tous les deux, couchés sous la moustiquaire."44 Une communauté à deux (qu'il s'agisse d'homme-loup et femme-truie ou d'une femme et d'une truie) ne peut rester isolée sous le réseau d'une moustiquaire, surtout lorsque cette figure est intimement liée au premier passage de La Condition humaine d'André Malraux, où Tchen occupe une position tout aussi perverse que celle de la femme-truie: pris dans une intense communauté à deux avec le corps de sa victime virtuelle, l'assassin de Malraux doit briser l'isolement de celui qu'il va tuer, ce qui l'exposera à son tour au monde à l'extérieur de la chambre. Pour abattre l'homme, c'est-à-dire le rendre chair morte, Tchen veut d'abord le ranimer car il lui est impossible de tuer un corps dormant; pris aux affres d'un désir multiple et vacillant entre deux espaces, intérieur et extérieur, il exprime l'indécision avec un fort devenir-assassinat et l'assassinat avec un devenir-indécision. ${ }^{45}$

La femme-truie est aussi indécise face au réseau d'Internet. Elle y est attirée dans l'espoir de rentrer en contact avec sa mère, espoir que le lecteur sait vain, car il a compris que le moi actualisé par la mère est éphémère et sans signification. Le problème de la femme-truie, vis-à-vis de sa mère et donc des origines de sa propre identité, est similaire à celui d'un Lorenzaccio ou d'un cybernaute:

[L]es participants à Internet, par exemple dans le cadre d'un Users Group, n'ont pas d'autre visage que ceux qu'ils se créent eux-mêmes, et ainsi pas

\footnotetext{
${ }^{42}$ Darrieussecq, p. 128.

${ }^{43}$ Darrieussecq, p. 132.

${ }^{44}$ Darrieussecq, p. 131.

${ }^{45}$ André Malraux, La Condition humaine (Paris: Gallimard, 1946), p. 7. Et bien entendu, cet acte qui est en passe de se réaliser chez Malraux rappelle celui qui est déjà en train dans la condition humaine-porcine de Darrieussecq.
} 
de "moi" assignable. C'est la dissolution du Visage comme identité dure et infranchissable: "on the Net, nobody knows you're a dog" ironisait une caricature du New Yorker. ${ }^{46}$

Truismes, comme roman-rhizome ou texte sans début ni fin, n'est qu'une série de faux départs. Les tentatives d'actualisation, de puiser une forme concrète dans l'écriture de cochon du devenir-œuvre, sont sans cesse minées par l'appel de l'intertexte. Qui la prend pour une truie en fin de compte? On s'intéresse à elle dans la mesure où elle se donne aux autres; on prend plaisir à toucher son corps comme un lecteur prendrait plaisir à aborder le texte lisible. C'est son corps pulpeux, son objectivité, qu'on adore chez elle, quelque forme qu'elle imagine avoir. Son identité-truie est une façon d'exister virtuellement, c'est-à-dire d'être hors d'elle-même. On ne trouve dans les traces laissées par la femme-truie qu'une identité virtuelle, que Mireille Buydens décrit comme un "curieux portrait [...] qui ne représente que mon désir en acte". ${ }^{47}$

C'est bien le portrait d'un désir en acte qui se dresse dans le dernier paragraphe de Truismes, et c'est un désir de dissolution qui marque, non pas la fin du texte, mais la volonté du texte d'exister ailleurs que sous les contraintes de sa forme immédiate, dans l'infini de l'intertexte: "J'essaie de faire comme me l'avait montré Yvan, mais à rebrousse-poil de ses propres méthodes: moi c'est pour retrouver ma cambrure d'humain que je tends mon cou vers la Lune."48 Il s'agit donc de retrouver sa cambrure d'humain par l'écriture dans sa dimension multiple, le scriptible, car la virtualisation, ainsi que le conçoit Pierre Lévy, exprime "la poursuite d'une hominisation continuée". ${ }^{49} \mathrm{La}$ méthode d'Yvan consistait à maîtriser l'actualisation de l'identité plurielle, sous forme de truie ou de femme; par opposition, retrouver sa cambrure d'humain serait plutôt un acte d'abandon au lecteur. On tend son cou pour s'offrir à la guillotine ou à la potence, mais au lieu de se suicider, la femme-truie se fait texte scriptible, et la Lune, avec son "l" majuscule, remplace dans le miroir - leitmotiv du texte - l'image de la multiple identité du corps en devenir par le visage de celui qui désormais tient la clé de son interprétation: le corps de la femme-truie se confond en fin de compte avec celui du lecteur.

Ainsi cette dernière image est-elle celle de la virtualisation de la protagoniste et de son monde: "Dans le cyberespace, mon visage et mon âme doivent sortir par mes doigts, devenir extériorité pure, surface lumineuse qui se donne à voir: le monde vrai n'y est qu'un hypothétique noumène et [...] je n'ai guère de substance au-delà de mon apparence." ${ }^{50}$ Malgré ses tentatives pour s'actualiser et prendre la forme de l'œuvre que le lecteur tient entre ses mains, la pluralité de la femme-truie se dissout toujours et à jamais dans la multiplicité du virtuel:

\footnotetext{
${ }^{46}$ Buydens, p. $62 . \quad{ }^{47}$ Buydens, p. 62.

${ }^{48}$ Darrieussecq, p. $158 . \quad{ }^{49}$ Lévy, p. 69.

${ }^{50}$ Buydens, p. 62.
} 
Il y a donc une perception deleuzienne d'Internet comme lieu de dissolution du moi, pour autant que je m'y donne un être changeant et multiple, c'està-dire que j'utilise la liberté offerte pour me perdre sans cesse dans un devenir-imperceptible: il ne s'agit donc pas en fin de compte de me présenter comme un autre (ce qui serait remplacer un moi par un moi), mais, au travers des visages changeants, comme un "superjet", c'est-à-dire un point de vue provisoire sur le monde et une sinueuse déclinaison d'affects. ${ }^{51}$

Ou, pour prendre une citation de Marie Darrieussecq, tiré d'un autre roman intitulé Naissances des Fantômes: "Ecrire c'est être entre deux mondes, là où rien n'est certain mais où tout est possible, où circulent les fluides, les sensations.."52

University of Newcastle, NSW

\footnotetext{
${ }^{51}$ Buydens, p. 62-63.

${ }^{52}$ Marie Darrieussecq, Naissances des Fantômes (Paris: Gallimard, 1999), 4ème de couverture.
} 